Rev. Elev. Méd. vét. Pays trop., 1965, 18, 3 (317-320).

\title{
Note sur l'aménagement des bas-fonds Malgaches pour la production fourragère*
}

\author{
P. GRANIER
}

\begin{abstract}
RÉSUMÉ
Les Baiboas des vallées du nord-ouest de Madagascar et les bords des thalwegs des hauts plateaux, sont actuellement peu ou mal utilisés.

Pour chaque cas, l'auteur décrit les techniques d'aménagemeni susceptibles d'être vulgarisées, ainsi que les plantes préconisées survant les conditions écologiques locales et les lypes d'exploilation recommandés, selon la spéculation recherchée (lait ou viande).

L'auteur insıste sur les possibilités de Brachiaria mutica, et de Stylosanthes gracilıs comme plantes de pâfurage, el de celles de Pennisefum purpureum el de Tripsacum laxum comme plantes de coupe.
\end{abstract}

Par suite du mode d'élevage pratıqué à Madagascar les bas-fonds n'ont pas été aménagés pour l'alimentation du bétall. La présente note a pour objet l'étude des possibilités offertes par ces terrains dans la zone sédimentaire de l'ovest ef sur les plateaux.

Les deux régions sont totalement différentes tant des points de vue physique, climatologique, phytogéographique, qu'économique ef social, aussi les techniques, les modes d'exploitation et les spéculations envisagés sont particuliers à chacune de ces régions.

\section{I. - Les bas-fonds de l'ouest.}

La zone sédimentaire de l'ovest comporte des superficies immenses de terres, dénommées «Baiboa », c'est-d̀-dire, inondées, alluvionnées, pendant la saison pluvieuse.

Les baiboas sont caractérisés :

- par leur richesse considérable ; constifuée par des alluvions profondes, bien minéralisées, à capacité d'échange élevée. Le $\mathrm{pH}$ oscille entre 5,2 et 6,8 . Seule, la matière organique est insuffisante et descend parfois jusqu'à 5 ou 6 p. 100.

* Communication présentée au IX Congrès International des pâturages. Sao-Paulo (Brésil), 7-20 Janvier 1965.
- par leur approvisionnement en eau, suffisant pour permettre la croissance des plantes pendant la saison sèche.

Ces terres souvent difficiles d'accès, nécessitent des défrichements coûteux qui sont rentabılisés par des cultures riches (tabac, coton, riz de luxe). Mais il existe aussi des zones marécageuses trop profondes, ou exondées trop tard pour être cultivées, et seule une culture fourragère pérenne et supportant l'inondation peut y être envisagée. D'autre part, l'élevage ne peut pas être éliminé de ces zones parce que :

- La traction animale demeure nécessaire pour certains travaux ef pour l'intégration des cultures riches dans la structure sociale actuelle.

- Ce sont des zones d'élevage et d'embouche, et seuls les baiboas peuvent permettre la survie du bétail, pendant la saison sèche qui dure d'avril à octobre (pluviométrie de 850 à $1.100 \mathrm{~m} / \mathrm{m}$ ). - Les engrais minéraux ne suffisent pas pour maintenir les rendements ef le fumier animal représente une richesse considérable et seul permet de pallier l'insuffisance en matière organique.

- Le développement de la production laitière ne pourra se faire dans ces régions à longue saison sèche que si les laitières disposent de fourrage" vert toute l'année. 
L'aménagement de ces zones consisterait essentiellement dans le défrichement, parfois sommaire, des terres et le bouturage d'herbe de para.

Cette graminée, Brochiaria mutica (Stapf) a été introduite dans l'ouest malgache en 1954.

La présente étude a pour objet de montrer la simplicité des façons culturales, la rusticité du Brochiorio et ses possibilités.

10 Façons culturales : elles ont pour but de permettre le bouturage, mais aussi d'éviter la concurrence de la végétation spontanée qui dans ces terrains profonds, brûle difficilement, est abondante et vigoureuse (cyperacées, mimosées, nymphées, palmiers, grandes graminées...).

a) Dans les zones marécageuses, le para est planté en saison sèche, à la décrue, après un piétinage du sol effectué avec un tracteur à rovescages, ou avec un troupeau de bœufs (comme dans la riziculture malgache).

b) Sur terrains humides, mais non marécageux, il faut préparer les terrains avant les pluies (labour) et attendre que le sol soitsuffisamment imbibé pour placer les boutures enracinées longues d'un mètre dans un sillon et les recouvrir légèrement de terre.

Dans un terrain encore submergé, on plante la bouture verticale avec quelques feuilles dans l'air ; dans un terrain non submergé, on place la bouture horizontalement avec un nœud dépassant. Parfois, un passage du tracteur à rovescages suffit pour enraciner les boutures.

$2^{\circ}$ Rusticité du Brachiaria mutica : la végétation spontanée piétinée meurt parce que la terre se dessèche et se craquèle, mais la bouture de para donne le premier mois un stolon d'une trentaine de centimètres, puis à partir du pied-mère 8 d̀ 9 stolons en moyenne se développent et d̀ trois mois atteignent deux mètres de lang. Les nœuds s'enracinent et les racines descendent dans les failles à la recherche de l'humıdité. Au mois de décembre, à la montée des eaux, les stolons sont suffisamment longs pour flotter et venir fleurir à la surface. A la décrue suivante le terrain est recouvert d'un tapis d'herbe sur un mètre d'épaisseur qui peut être pâturé.

Dons les zones marécageuses, le para planté au début des pluies se laisse envahir par la végétation spontanée.

Le pâturage ainsi aménagé est pratiquement définitif. Dans les essals effectués dans l'ouest de 1956 à 1962, le para a résisté aux feux courants ef au surpâturage pendant des années consécutives. Après 6 mois de saison sèche, la mise en: défens d'un témoin surpâturé, a permis de constater que, sur les sols ou la nappe phréatique est proche, sa croissance est continue. Dans le carré témoin, les souches rasées au niveau du sol, ont donné des stolons de $35 \mathrm{~cm}$ en 10 jours, malgré la persistance de la sécheresse.

30 Possibilités : les analyses de foin de para ont donné des résultats satisfaisants - valeur fourragère $-0,38 \mathrm{U}$. $F$.

Les rendements sont importants, ils représentent suivant les sols 65 à $100 \mathrm{t} /$ ha en vert, dans des régions où le rendement des pâturages de savane est de l'ordre de 10 t/ha.

L.'appétıbilité est excellente et le para résiste parfaitement au pâturage et au piétinement.

En zone marécageuse, 6 ha de para ont assuré une croissance normale pendant la saison sèche à un troupeau donnant une charge d̀ l'ha de 7 unités bétail de $250 \mathrm{~kg}$ de moyenne.

En conclusion, Brochioria mutica est une graminée qui se bouture facilement avec une façon culturale simple, elle reprend vigoureusement, couvre rapidement le sol, apporte beaucoup de matière organique, résıste très bien à la sécheresse, aux feux courants, constitue un pâturage très recherché en saison sèche et très appété du bẻtall.

Il offre l'avantage de créer un pâturage permanent à la portée du paysan malgache, sa culture s'apparente à celle du riz, elle nécessite peu d'entretien et du fait de l'inondation la rotation. et la mise au repos s'effectuent natureilement, le pâturage n'étant disponible qu'en fin de saison sèche.

C'est une graminée très adaptée par la charge à I'ha qu'elle tolère, pour l'aménagement des zones d'embouche, des zones de parcours de saison sèche, et des' grandes zones d'élevage d'autanł qu'en terrain non inondés elle s'associe très bien avec le kudzu (Pueraria javanica), Melinis minuflora, Stylosanthes gracilis, Centrusema pubescens...

\section{II. - Les bas-fonds de la région centrale.}

Dans la région des Hauts-Plateaux et du Moyen Ovest un effort considérable est fait actuellement 
pour orienter l'élevage vers la production laitière à partir de croisements avec les races Normande, Friesland et Sahiwal.

Les pâturages naturels sont insuffisants pour assurer une production zootechnique pendant les trols derniers mois de la saison sèche (durée 6 mois) et la production laitière demande une alimentation équilibrée que seules des cultures fourragères à contre-saison pourront fournir. D'autre parti, la pâture d'association graminées! légumineuses durant les 3 mois qui précèdent la saison de reproduction, ne peut qu'améliorer la qualité de la ration et influencer la fécondité et la lactation des vaches.

Le facteur « equ» étant limitant, seules les terres du bas-fond, terres riches et irrigables, peuvent permettre le développement de la production laıtière.

Dans ces zones, une érosion intense et ancienne a 'modelé un faciès bien particulier fait d'une succession de collines ef de bas-fonds. Ces basfonds, dont la pente va de 2 à 4 p. 100 , présentent l'intérêt d'être drainables et de posséder des nappes phréatıques perchées, situées en tête du thalweg. L'aménagement comporte, soit un simple drainage, soit essentiellement :

- La récupération de l'eau en tête de basfond par un barrage en terre.

- Le drainage du bas-fond - les drains suivant la pente naturelle et se dirigeant vers les colluvions plus basses.

- L'utilisation de l'eau pour l'irrigation de la plus grande surface possible de colluvions.

L'irrigation est alternée pour permettre les façons culturales, les pacages, les temps de repos, et de décaler dans le temps descycles végétatifs. En outre, un rythme alterné de submersion/ assèchement est favorable à l'activité microbienne du sol, à la fixation d'azote donc à une meilleure utilisation de la fumure organique ( $30 \mathrm{t} / \mathrm{ha}$ ).

Trols parties sont à distinguer dans ces basfonds :

- Lo pente : elle ne peut pas être labourée, elle est aménagée avec une culture de Kudzu ou de centrosema en trous creusés en quinconce le long des lignes en courbe de niveau, ou bien reboisées.

- Le marécage: après drainage, il permet l'installation des rizıères et de pâturages d'herbe de para en association ovec la végétation spon- tanée (panicées, oryzées, cypéracées, légumineuses).

- Les colluvions : sont les plus importantes, parce qu'elles peuvent avec une fumure organique de l'ordre de 30 t/ha, et une irrigation hebdomadaire, fournir une grande quantité d'unités fourragères étalées dans la saison sèche.

Un fait domine, c'est leur hétérogénéité ; de la pente vers le marécage, on a un gradient d'humidité et d'acidité, et on y rencontre parfois des gleys et des tourbes qui nécessitent un drainage supplémentaire ou des cultures sur billons.

Les meilleurs résultats sont donnés actuellement par les graminées c̀ haut rendement et les associations légumineuses/graminées.

\section{1) Les graminées à hout rendement.}

a) Pennisetum purpureum, variété «à collet rouge $》$.

Plantée par boutures à 4 nœuds, en terrain fumé, au mois de mars, pour pouvoir obtenir 4 coupes par an, cette graminée présente l'intérêt de permettre 2 coupes en saison sèche. Le rythme des coupes s'établit comme suit :

$$
\begin{aligned}
& \text { 1re coupe - juillet } \\
& 2^{\mathrm{e}} \text { coupe - octobre } \\
& 3^{\mathrm{e}} \text { coupe - décembre } \\
& 4^{\mathrm{e}} \text { coupe - fin janvier. }
\end{aligned}
$$

Les rendements, lorsque les souches sont coupées alors qu'elles atjeignent $1.50 \mathrm{~m}$ de hauteur, sont de 40 t par coupe, soit 160 t par an ef par ha.

La moyenne des rendements est de $6 \mathrm{~kg}$ par pied.

La valeur fourragère étant de 0,10 U.F. (vert), on obtient donc, avec un ha de Pennisetum, fumé après chaque coupe, $16.000 \mathrm{U}$. F. par an.

b) Trypsacum laxum ou « guatemala grass». Cette graminée, bien que cultivée dans les mêmes conditions que la précédente et dans le même but, s'en éloigne par sa composition, son cycle végétatif et son rapport feuilles/tiges.

Ceste maydée dont les feuilles sont très engainantes ef nasssent très près du sol, donne des pieds de $5 \mathrm{~kg}$ de moyenne, qui peuvent être consommés en totalité par le bétail sans nécessiter un passage au hache-paille. Elle est moins riche en lignine et cellulose que lo précédente et plus riche en matières protéiques. 
Son coefficient d'encombrement permet d'obtenir une ration plus équilibrée qu'avec Pennısefum purpureum. Malheureusement, sa croissance est plus lente et dans les mêmes conditions que Penniselum, elle permet 3 coupes au lieu de 4 ; chaque coupe donne également 40 t/ha, soit par an $120+$ avec 1 coupe en saison sèche (juillet).

\section{2) Les légumineuses.}

Stylosanthes gracilis contrairement d̀ ce que I'on observe en Afrique, germe très facilement à Madagascar. Il supporte bien le pâturage, qui permet de le propoger naturellement par les excréments du bétail.

Bien que donnant de bons résultats sur terres de plateaux ( $43 \mathrm{t} / \mathrm{ha}$ ), il a permis d'installer des pâturages de bas-fonds, où avec une irrigation minimale, il a des rendements au bout d'un an, de $70 \mathrm{t} / \mathrm{ha}$.

II offre l'intérêt de supporter très bien les associations avec Brachiaria, Melinis et Chloris. En association, on peut réduire les semis de Stylosanthes à $2,5 \mathrm{~kg} / \mathrm{ha}$.

\section{3) Les associations.}

En colluvions irriguées, les rendements sont qugmentés et surtout les affouragements équilibrés avec une association :

\section{Mucuno utilss/Pennisetum purpureum}

Les boutures de Pennisetum sont mises en terre, et lorsque la première feville atteint une trentaine decentimètres, Mucuno est semé entre les lignes. Sa croissance est rapide et les deux plantes s'associent parfaitement.

Dans les régıons à vocation non essentıellement rizicole il est donc actuellement possible d'améliorer le milieu pour obtenir la quantıté d'unités fourragères nécessaires à la production laitière pendant toute l'année. Ce n'est qu'à cette condition, qu'est rendue possible, l'introduction de races améliorées (Friesiand, Normande) qui rentabiliseront les investissements «travaux » à la portée du paysan malgache.

\section{SUMMARY}

Nole on the management of low-lying ground in Madagascar for the production of forage

The Baibon of the North-West valleys of Madagascar and the heads of the high-lands thalweg are actually few or badly used.

For each case the author describes the management technics capable of popularization, the plants cracked up according to the local ecological condifrons, the cultivation types recommended according to the searched speculation (milk or meat).

The author dwells on the possibilities of Brachiaria mutica and Stylosanthes gracilis as grazing plants and of Pennısetum purpureum and Tripsacum laxum as plants for cutting.

\section{RESUMEN}

\section{Nota sobre el acondıcionamiento de los bajos fondos malgachos} para la produccion forrajera

Los Baiboas de los valles del N-O de Madagascar y las cabeceras de los thalwegs de las altas mesetas, estan actualmente poco o mal utilizadas.

Para cado uno de los casos el autor expone las técnicas de acondicionamiento susceptibles de ser vulgarizadas, asi como las plantas preconizadas por las condiciones ecológicas locales y los tipos de explotación a recomendar, según la especulación buscada (leche o carne).

El autor insiste sobre las posibilidades de la Brachırio mutica, y de Stylosanthes gracilis como plantas de pasto, $y$ las de Pennisetum purpureum, y del Tripsacum laxum como plantas de corte.

\section{BIBLIOGRAPHIE}

\section{GRANIER (P.). - L'herbe de Para dans la SERVICE DE L'ÉLEVAGE DE MADAGASCAR. - Province de Majunga. Bulletin de Madagascar, 1962, (191) : 353-61. Rapports' annuels 1961 et 1962.}

